\title{
Prolapsing giant right atrial myxoma in a young male presenting as pulmonary embolism
}

\author{
Bhupinder Singh ${ }^{1}$, Rajiv Kumar Gupta ${ }^{2}$, Rohit Tandon ${ }^{1}$, Ankita Soni ${ }^{2}$, Harpreet Kaur ${ }^{2}$, \\ Bishav Mohan ${ }^{1}$, and Gurpreet Wander ${ }^{1}$ \\ ${ }^{1}$ Dayanand Medical College and Hospital Department of Cardiology \\ ${ }^{2}$ Dayanand Medical College and Hospital
}

January 30, 2022

\begin{abstract}
A 22-year male presented with complaints of dyspnea. Multimodality imaging revealed a polypoidal right atrial mass with submassive pulmonary embolism. The patient underwent urgent surgery. The pathological examination confirmed it as cardiac myxoma. Cardiac myxoma, a most common primary cardiac tumor, is commonly found in the left atrium. The right atrium is an uncommon site and the usual mode of presentation is the tumor or thrombus embolisation to the pulmonary circulation.

Prolapsing giant right atrial myxoma in a young male presenting as pulmonary embolism

Bhupinder Singh $^{1 *}$, Rajiv Kumar Gupta ${ }^{2}$, Rohit Tandon ${ }^{1}$, Ankita Soni ${ }^{3}$, Harpreet Kaur ${ }^{3}$, Bishav Mohan ${ }^{1}$, Gurpreet S Wander ${ }^{1}$.

${ }^{1}$ Department of Cardiology, Dayanand Medical College and Hospital, Ludhiana, Punjab, India.

${ }^{2}$ Department of cardiothoracic surgery, Dayanand Medical College and Hospital, Ludhiana, Punjab, India.

${ }^{3}$ Department of Pathology, Dayanand Medical College and Hospital, Ludhiana, Punjab, India.
\end{abstract}

\section{* Corresponding author:}

Dr. Bhupinder Singh,

Dayanand Medical College and Hospital, Udham Singh Nagar, Ludhiana 560069, India.

Tel.: +918427821837, Email: dr_bhupinders@yahoo.in

Fax number: none

Short Title: Prolapsing right atrial myxoma

Disclosures: The authors have no financial interests or conflict of interest regarding the content.

Data Availability Statement: The data that support the findings of this study are available in the supplementary material of this article.

Word count: 220

Abstract: A 22-year male presented with complaints of dyspnea. Multimodality imaging revealed a polypoidal right atrial mass with sub-massive pulmonary embolism. The patient underwent urgent surgery. The 
pathological examination confirmed it as cardiac myxoma. Cardiac myxoma, a most common primary cardiac tumor, is commonly found in the left atrium. The right atrium is an uncommon site and the usual mode of presentation is the tumor or thrombus embolisation to the pulmonary circulation.

A 22-year previously healthy male presented with complaints of progressive dyspnea of 1-week duration. On examination, he had tachypneic, tachycardia, and peripheral oxygen desaturation. 12-lead electrocardiogram showed sinus tachycardia, complete right bundle branch block, and S1Q3T3 pattern. Chest roentgenogram showed wedge-shaped opacity in the right upper-middle zone. Transthoracic echocardiogram (panel A, video) revealed dilated right-sided chambers, and a large, polypoidal, right atrial mass $(\mathrm{RA}$; measuring $6.0 \times 3.1 \mathrm{~cm})$ prolapsing into the right ventricle with each cardiac cycle. Contrast-enhanced computerised tomography of thorax confirmed the presence of RA mass attached to the posterior wall of RA, and filling defects in MPA (panel B) extending to the right and left pulmonary arteries (right $>$ left). The patient underwent urgent surgical excision (video) of RA mass and pulmonary embolectomy. The pathological examination (panel C, D) confirmed cardiac myxoma. The patient is doing well at 12-months of follow-up with no recurrence.

Cardiac myxoma is the most common primary tumor of the heart. The left atrium is the most common site for cardiac myxoma while $15-20 \%$ of cases originate in RA. ${ }^{1}$ The clinical presentations depend on the site, size, mobility, and friability of the cardiac mass. ${ }^{2}$ Cardio-embolic events occur in around $30-40 \%$ of the cases. ${ }^{2}$ The RA myxoma complicated with pulmonary embolism is usually due to tumor embolisation, although thrombotic embolisation has also been reported less frequently. ${ }^{3}$

\section{References:}

1. Yang TY, Tsai JP, Chang CH, Kuo JY, Hung CL. Giant right atrial myxoma with pulmonary trunk dislodgement causing intermittent tricuspid obliteration and clinical manifestations of right heart failure. Echocardiography. 2011 Oct;28(9):E183-6.

2. Reynen K. Cardiac myxomas. N Engl J Med. 1995 Dec 14;333(24):1610-7.

3. Fabijanić D, Rudež I, Radić M, Unić D, Barić D, Kardum D. Pulmonary embolism due to the right atrial thrombus mimicking atrial myxoma. Chin Med J (Engl). 2010 Sep;123(17):2483-5.

\section{Figure legends:}

(Panel A) Transthoracic echocardiogram revealing dilated right-sided chambers, an echogenic, polypoidal right atrial mass (measuring $6.0 \times 3.1 \mathrm{~cm}$ ) prolapsing into the right ventricle. (Panel B) Contrast-enhanced computerised tomography of thorax showing filling defects in main pulmonary artery extending to the right and left pulmonary arteries (right $>$ left) with segmental and sub-segmental arterial involvement. (Panel C) Gross examination of the mass showing glistening-yellow cut surface with areas of calcification (arrow). (Panel D) Photomicrograph showing abundant myxoid matrix with singly scattered stellate cells suggestive of atrial myxoma.

Video: Video showing sequential conglomeration of multimodality imaging, peri-operative findings, and pathological photographs. 

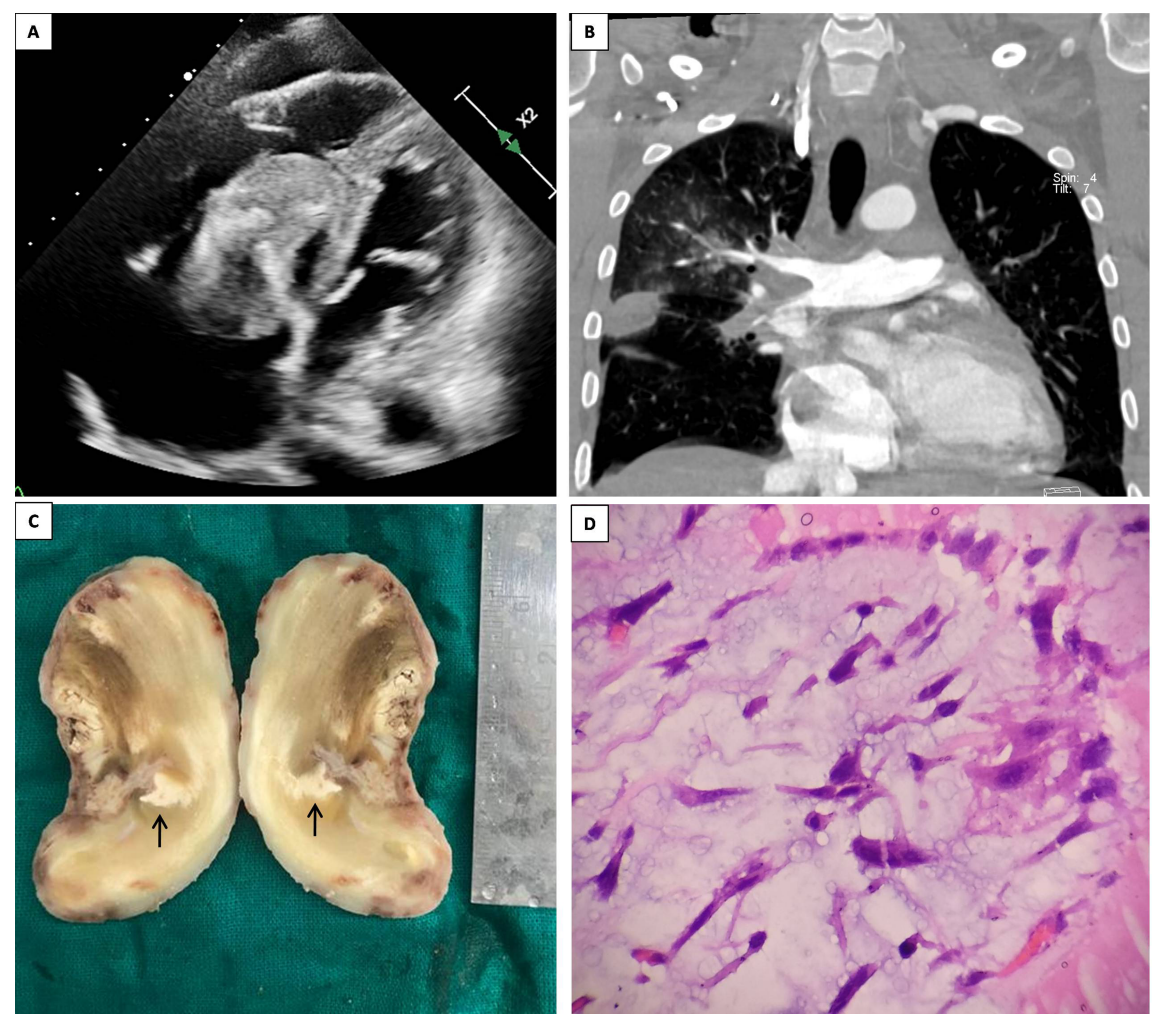